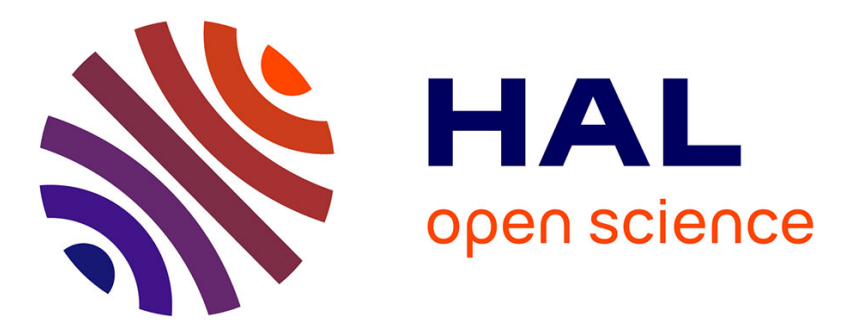

\title{
A level set approach for the simulation of the multipass hybrid laser/GMA welding process
}

Olivier Desmaison, Michel Bellet, Gildas Guillemot

\section{To cite this version:}

Olivier Desmaison, Michel Bellet, Gildas Guillemot. A level set approach for the simulation of the multipass hybrid laser/GMA welding process. Computational Materials Science, 2014, 91, pp.240-250. 10.1016/j.commatsci.2014.04.036 . hal-01004625

HAL Id: hal-01004625

https://hal-mines-paristech.archives-ouvertes.fr/hal-01004625

Submitted on 11 Jun 2014

HAL is a multi-disciplinary open access archive for the deposit and dissemination of scientific research documents, whether they are published or not. The documents may come from teaching and research institutions in France or abroad, or from public or private research centers.
L'archive ouverte pluridisciplinaire HAL, est destinée au dépôt et à la diffusion de documents scientifiques de niveau recherche, publiés ou non, émanant des établissements d'enseignement et de recherche français ou étrangers, des laboratoires publics ou privés. 


\title{
A Level Set Approach for the Simulation of the Multipass Hybrid Laser / GMA Welding Process
}

\author{
Olivier DESMAISON, Michel BELLET, Gildas GUILLEMOT \\ Mines ParisTech, CEMEF - Centre de Mise en Forme des Matériaux, \\ CNRS UMR 7635, CS 10207, 06904 Sophia Antipolis, France \\ olivier.desmaison@mines-paristech.fr,michel.bellet@mines-paristech.fr, \\ gildas.guillemot@mines-paristech.fr
}

\begin{abstract}
A new 3D finite element model, developed in a level set approach, is proposed to model hybrid gas metal arc / laser welding in multipass conditions. This heat and mass transfer model couples the resolution of the heat conservation equation, the momentum and mass conservation equations and the weld bead development. The arc welding total power is divided in two parts: one corresponding to the energy transferred to the fusion zone by droplets of melted filler material, the rest being transferred to the workpiece by the surrounding plasma. The droplets energy input is modelled as a volumetric heat source. Regarding the heat surface fluxes associated with plasma and laser heating, the "Continuum Surface Force" approach is used to model them as volumetric heat sources concentrated in the immediate neighbourhood of the metal-gas interface. A resolution scheme, consisting in ignoring high velocity fluid flow in the fusion zone, through the use of an augmented liquid viscosity, is proposed and discussed. Accordingly, the liquid thermal conductivity is enhanced to result in a realistic heat transfer to the workpiece. The formation of the weld bead is obtained through the introduction of a source term in the mass conservation equation, and the application of the normal component of surface tension forces, proportional to the mean curvature of the metal-gas interface. This approach is proposed to reduce computation time. The resolution scheme is applied to the simulation of hybrid welding of 18MND5 (ASME SA 533) steel grade. Results are compared to experimental measurements and observations operated in conditions close to industrial ones. The influence of the enhancement applied to the liquid conductivity coefficient is shown and discussed. A strong sensitivity evolution is demonstrated when varying it from the physical value to the value proposed in the welding literature. As proposed, the simplified resolution scheme leads to a good estimation of the weld bead surface development. Nevertheless, there are still noticeable differences with the whole set of experimental results that are discussed and can be explained by model limitations and insufficient knowledge of material and interfacial properties.
\end{abstract}

Keywords: hybrid multipass welding, level set approach, heat and mass transfer model, arc laser 


\section{Introduction}

The large use of welding in heavy industries has lead to continuous efforts in developing numerical simulation approaches in the last twenty five years. The understanding of weld pool dynamics and solidification during gas metal arc welding (GMAW) is of great importance to master the spreading of fusion and heat affected zones through the weld piece, the shape of weld beads, and the formation of deformations and stresses in the neighbourhood of the fusion zone. Indeed, these local stresses may lead to hot tearing (solidification cracking) for some critical alloys and welding conditions (process conditions and local design of welded components). This highlights the need for accurate numerical modelling, to improve and better control welding processability. Recently the efficiency of GMAW processes has been enhanced through the coupling with a laser beam. Located ahead of the torch, this new heat source provides additional energy to the workpiece. The resulting weld pool spreading enables to supply more material, to mix it more homogeneously and consequently to weld better and faster.

Since the development of 2D-axisymmetric stationary models for gas tungsten arc welding (GTAW) processes at the beginning of the 1990's [1, 2, 3] significant progress has been made. Recent models are now able to realistically simulate GMAW processes taking into account the main phenomena inside both the arc plasma and the weld pool $[4,5,6]$. Consequently two modelling approaches spring up. The first one considers an explicit modelling approach of heat and mass transfer: electromagnetic fluxes inside the arc plasma are computed. They are next taken into account as the main heat source $[4,5,7,8]$. The second approach, such as the one presented in the present paper, uses analytical models to simulate the heat source and material supply: arc plasma phenomena are not explicitly modelled. Over the years, the simplified Goldak approach and the laminar flow analysis in the weld pool have tended to be replaced by more physically coupled models including a Gaussian surface heat flux and turbulent flows without any enhanced viscosity [9, 10, 11, 12]. However the modelling of turbulent flows has only been proposed in steady conditions, for a coordinate system moving with the heat source. No valuable modelling in unsteady or transitory conditions seems to be proposed. Moreover, since it has been experimentally [13] and numerically [14] proved that Lorentz forces and Marangoni effects are the two main driving forces for weld pool hydrodynamics, these forces appear in almost all new papers. Regarding free surface deformation, surface tension and arc pressure are also taken into account in the minimization of the surface energy $[14,15,16]$. Lastly, buoyancy forces can no longer be neglected since it has been proved they have a great influence in horizontal GTA welding [17]. Because of the improvements in hybrid welding modelling and the research focus on keyhole phenomenon, these numerical methods have been carried over to laser/GMAW modelling [18, 19, 20, 21, 22]. As a result, the numerically obtained bead shapes better fit the experimental ones. Nevertheless, only very few models consider the entirety of the previous phenomena. Kumar and Debroy [14], for instance, propose an advanced GMAW model in 3D which remains stationary and laminar. Recently Wang et al. [23] have developed a 3D transient model for laser keyhole welding through the volume of fluid (VOF) approach to study the temperature field evolution. Others, such as Traidia and Roger [8], could model the effects of a pulsed GTA welding on the weld pool geometry through a 3D transient model. The results presented in those both papers are promising but, inherently for those processes, no material supply is taken into account.

A transient three dimensional finite element model developed to study multipass welding is presented in this paper. 3D P1+/P1 (velocity/pressure) tetrahedral elements are used. The model includes the workpiece and a surrounding gaseous domain. The electrode, the arc plasma and the droplets are not modelled. Nevertheless theirs influences on weld bead development are modelled as boundary conditions at the gas-metal interface or as source term inside the weld pool. The gaseous domain consists of the arc plasma region over the fusion zone and of the air where the weld joint is to be formed. Therefore a Eulerian approach is used in which the interface between the metal and the surrounding gas is defined by a level set function. Because the arc plasma is not modelled, the heat transferred from the arc plasma to the piece is defined as a surface boundary condition according to a Gaussian distribution. In the level set framework, this surface heat flux is converted into a volumetric heat source through the "Continuum Surface Force" method [24]. The laser heat source is also modelled in this way. It should be mentioned that in the present paper the study is restricted to unfocused laser beam, which means that keyhole effects are clearly out of the scope of the model developed. Unfocused and moderate power laser is used here as an auxiliary heat source, the role of which is essentially to enlarge the welding pool. In addition a third volumetric heat source is implemented to model the heat supply associated with the impingement of molten droplets into the fusion zone [10, 14, 15, 16]. Moreover, for the mass input of these molten droplets, a source term is added to the right hand side of the mass conservation equation for certain liquid finite elements in the fusion zone [25]. A compressible non steady-state Navier-Stokes equation is then solved taking into account surface tension forces (the normal component associated with mean curvature) and gravity. In order to limit as much as possible the computational time, this Navier-Stokes equation is solved with an enhanced viscosity. Proceeding this way, the focus is set on weld bead formation, rather than fluid flow in the 
fusion zone. Next the obtained velocity field is used to solve the transport of the level set function that is the updating of the gas-metal interface. The finite element mesh is regenerated periodically to fit to the fusion zone and the interface displacements. This model aims to predict the weld bead development in a multi-pass welding process.

The paper will present first the mathematical formulation of the finite element problem. The three main governing equations - energy, mass and momentum equations - will be detailed, as well as the boundary conditions and source terms in the context of hybrid arc-laser welding. Secondly the model application to the simulation of a multipass welding test in conditions approaching industrial conditions will be described. A part is proposed in order to demonstrate and discuss the influence of the enhancement applied on the thermal diffusivity coefficient on the weld pool geometry and temperature field. Lastly, the numerical results will be analyzed. The performance and efficiency of the proposed resolution scheme will be discussed.

\section{Numerical Model}

\subsection{Level Set Approach}

\subsubsection{Metal Gas Interface}

A domain including the weld piece and a gaseous domain in its neighbourhood (where the weld joint is expected to form) is defined and meshed initially. An Eulerian approach is developed to model the hybrid Laser / GMAW process as illustrated in Figure 1. At any time of the process, the gas-metal interface is identified thanks to the definition of a level set (LS) function, $\varphi$ corresponding to the signed distance of any point $M$ to this interface (Figure 2). It is proposed to use positive values of the LS function in order to identify the gas domain. Negative values of the LS function identify both liquid and solid metallic phases. The LS approach is proposed to overcome the numerical and modelling difficulties due to metal / chamfer contacts or to the contacts between successive passes in multipass welding process.

\subsubsection{Mixing Law}

The finite element mesh is used to discretize and solve the heat, mass and momentum conservation equations in the entire domain. In order to ensure the resolution of this set of equations, all material properties have to be averaged using the local value of the smoothed Heaviside function, $H(\varphi)$, as defined in the following equation.

$$
\begin{array}{ll}
H(\varphi)=0 & \text { if } \varphi<-\varepsilon \\
H(\varphi)=\frac{1}{2}\left[1+\frac{\varphi}{\varepsilon}+\frac{1}{\pi} \sin \left(\frac{\pi \varphi}{\varepsilon}\right)\right] & \text { if }|\varphi|<\varepsilon \\
H(\varphi)=1 & \text { if } \varphi>+\varepsilon
\end{array}
$$

where $\varepsilon$ is the half-thickness of the transition zone around the interface defined by $\varphi=0$ (Figure 2). The smoothed Heaviside function $H$ evolves from 0 to 1 in the thickness of the transition layer $[-\varepsilon:+\varepsilon]$. Any material property, $\alpha$, is then locally defined as an average of local gas and metal properties, $\alpha_{g}$ and $\alpha_{m}$ as:

$$
\alpha=[1-H(\varphi)] \alpha_{m}+H(\varphi) \alpha_{g}
$$

Using Eq.(2) on the whole mesh, the values of the material properties outside this narrow area correspond to the values in either the gas or the metal domain. They continuously evolve only in the narrow transition domain as a function of the signed distance to the interface, $\varphi$.

\subsection{Heat Transfer and Energy Equation}

The temperature field evolution during the welding process is calculated using a full resolution of the heat conservation equation. The non-steady state expression of the equation is:

$$
\rho C_{p}\left(\frac{\partial T}{\partial t}+\vec{v} \cdot \vec{\nabla} T\right)-\nabla \cdot(\lambda \vec{\nabla} T)=\dot{Q}
$$


where $\rho, C_{p}$ and $\lambda$ are the density, the specific heat and the thermal conductivity of the material, respectively. Vector $\vec{v}$ is the local fluid velocity to be considered (defined in $§ 2.4$.1. ). The right hand side $\dot{Q}$ is a volumetric heat source that will be detailed in the sequel.

\subsubsection{Material properties}

In order to take into account the latent heat associated with the solid-liquid phase change, $C_{p}$ in the previous equation denotes in fact an effective specific heat. It is calculated using Morgan's model [26] to approach the derivation of the specific mass enthalpy, $h$, with respect to the temperature $T$ :

$C_{p}^{t}=\frac{h^{t}-h^{t-\Delta t}}{T^{t}-T^{t-\Delta t}}$

where $t$ and $\Delta t$ are the current time and time step, respectively. The evolution of the specific enthalpy as a function of temperature is defined a priori for the entire temperature range. A program has been developed in order to calculate this evolution for a specific solidification path model. Thermodynamic equilibrium calculations are based on the software ThermoCalc (ThermoCalc SA) and the specific TCFE6 steel database. The Gulliver-Scheil solidification path model $[27,28]$ is proposed for the solid fraction evolution during cooling. Consequently the micro segregation model corresponds for all chemical species to a complete diffusion in the liquid phase and a non-diffusion in the solid phase which is appropriate to the rapid solidification encountered in the context of welding. By consequence the mushy zone is connected to the liquid fraction which is a function of the temperature. Thermal diffusivities in the solid phase are obtained from the literature [29, 30]. In the liquid phases, thermal diffusivities are considered as a constant value. In the mushy zone, which is in between the liquidus and solidus temperature, the local thermal diffusivities are deduced from a mixing law using the liquid fraction. Nevertheless an enhanced conductivity is used in the liquid phase in the case of the simplified resolution scheme presented above. This enhanced value is used, as often in the literature, to counterbalance the absence of a representative resolution for the hydrodynamics in the fusion zone. It tends to simulate the decrease of temperature gradient induced by fluid flows in the liquid pool.

\subsubsection{Heat sources in hybrid GMA / laser welding}

The local volumetric heat source $\dot{Q}$ is considered as the sum of several heat sources (Figure 3). The laser beam provides the first heat source encountered by the metal. A Gaussian condition is considered at the gasmetal interface (Figure 4) corresponding to a surface heat flux defined as:

$q_{L}=\frac{3 P_{L}}{K_{L} \pi R_{L}^{2}} \exp \left(-\frac{3 r^{2}}{R_{L}^{2}}\right)\left|\vec{u}_{L} \cdot \vec{n}_{L}\right|=\frac{3 P_{L}}{K_{L} \pi R_{L}^{2}} \exp \left(-\frac{3 r^{2}}{R_{L}^{2}}\right) \cos \left(\phi_{L}\right)$

where $r$ is the distance from the considered point to the direction of the moving laser beam. $R_{L}$ is the parameter of the Gaussian source and $P_{L}$ is the power of the laser beam. This surface heat flux, $q_{L}$, is applied for any distance $r$ lower than $R_{L}$. $K_{L}$ is the normalisation factor, equal to 0.95 . $\Phi_{L}$ is the angle between the normal $\vec{n}_{L}$ to the interface and the direction of the laser beam, $\vec{u}_{L}$.

The weld pool then develops due to two heat sources associated with the GMA electrode. The arc plasma provides first a surface heat flux $q_{p}$. There is a second heat input in the weld pool, associated with the succession of molten droplets impinging it. This second source will be defined in $\S 2.3$. The surface heat flux due to plasma transfer, $q_{p}$, is defined as an angular Gaussian condition [31] (see Figure 4):

$q_{P}=\frac{3 P_{P}}{K_{P} 2 \pi d^{2}(1-\cos \alpha)} \exp \left(-3\left(\frac{\beta}{\alpha}\right)^{2}\right)\left|\overrightarrow{u_{P}} \cdot \overrightarrow{n_{P}}\right|=\frac{3 P_{P}}{K_{P} 2 \pi d^{2}(1-\cos \alpha)} \exp \left(-3\left(\frac{\beta}{\alpha}\right)^{2}\right) \cos \phi_{P}$

where $\vec{u}_{P}$ is the unit vector between the tip of the electrode, $O$, and the point considered $M_{P}, d$ is the distance between points $O$ and $M_{P}, \beta$ is the angular coordinate of $M_{P}$ and $\Phi_{P}$ is the angle between the normal to the surface and the vertical direction. $P_{P}$ is the power of the heat source and $\alpha$ is the half-angle of the heat source geometry. $K_{P}$ is a normalisation factor expressed as:

$K_{P}=0.95+0.0347 \alpha^{2}$ 
The power of the plasma heat source $P_{P}$ is computed from an energy balance. A part, $P_{D}$, of the total GMAW torch power, $P_{G M A W}$, is used to melt the electrode of filler metal in the form of droplets. The sum of both heat powers $P_{P}$ and $P_{D}$ corresponds to the whole power of the GMAW process $P_{G M A W}$ :

$P_{\text {GMAW }}=\eta U I=P_{P}+P_{D}$

where $U$ and $I$ are respectively the tension and intensity applied to the GMAW torch, and $\eta$ is the global efficiency of the GMAW power source $(\eta<1)$. The power $P_{D}$ required to melt the electrode is related to the value of droplets temperature $T_{d}$, by the following expression:

$P_{D}=\left[h\left(T_{d}\right)-h\left(T_{0, w}\right)\right] \rho_{w}\left(T_{0, w}\right) v_{w} \pi R_{w}^{2}$

where $\rho_{w}\left(T_{0, w}\right)$ is the density of the filler wire material for its initial temperature $T_{0, w}$ considered as ambient temperature, $v_{w}$ its velocity and $R_{w}$ its radius. The plasma heat source $P_{P}$ is then computed as the difference between the total GMAW power, $P_{G M A W}$, and the electrode fusion power $P_{D}$.

Heat convection and radiation at the metal-gas interface correspond to the third surface heat flux, $q_{T}$. It is considered as the sum of fluxes corresponding to a Fourier boundary condition, $q_{T}^{d c}$, and to the StefanBoltzmann law application, $q_{T}^{S t}$. Consequently, $q_{T}$ is expressed as:

$q_{T}=q_{T}^{d c}+q_{T}^{S t}=h_{T}\left(T-T_{0}\right)+\varepsilon_{r} \sigma\left(T^{4}-T_{0}^{4}\right)$

where $h_{T}$ is the heat transfer coefficient, $\varepsilon_{r}$ the emissivity of the metal surface and $\sigma$ the Stefan-Boltzmann constant.

It should be observed that the heat sources $q_{L}, q_{P}$ and $q_{T}$ have to be imposed as three surface boundary conditions at the metal / gas interface. However, it is noticeable that the level set approach prevents to impose such specific surface conditions. Indeed, using the level set formulation, the interface is defined as the continuous surface corresponding to the zero-value of the level set function $\varphi=0$. This interface is not explicitly discretized and evolves through the meshed domain. Consequently it is not possible to impose directly the surface Neumann conditions corresponding to the preceding heat sources. It is proposed to use the Continuum Surface Force (CSF) method developed by Brackbill et al. [24] to transform these surface heat fluxes into volumetric source terms $[18,19]$. Considering the area $S$ of the gas-metal interface and a surface heat flux $q_{S}$, the whole heat transferred per unit time can be expressed as:

$P=\int_{S} q_{S} d \Gamma=\int_{V} q_{S} \delta(\varphi) d \Omega=\int_{V} \dot{Q}_{V} d \Omega$

where $V$ is the volume of the global model and $\delta$ is the derivative of the smoothed Heaviside function $H$ with respect to the level-set function. This function $\delta$ can be seen as a discretized Dirac function, which sharply evolves in the transition layer of thickness $2 \varepsilon$ around the interface and is null outside of this transition layer. Considering Eq.(11), the application of the surface heat flux $q_{S}$ onto the interface $S$ can be transformed into a volume heat source $q_{S} \delta(\varphi)$ corresponding to $\dot{Q}_{V}$, imposed in the transition layer defined by $|\varphi|<\varepsilon$. This method is applied to the three heat surface fluxes $q_{L}, q_{P}$ and $q_{T}$.

The impingement of molten droplets of filler metal in the weld pool corresponds to the last heat source to be considered. The filler metal melts at the electrode tip, and the droplets fall into the liquid bath with a high velocity. Lancaster [32] proposed to define a corresponding volume heat source in a cylinder below the torch, as illustrated in Figure 3. The expression of this volume heat source $\dot{Q}_{d}$ and extra details are presented hereafter in the Material supply paragraph (\$2.3.1. ) and (§2.3.2. ).

Finally, the total volume heat source applied on the whole mesh, $\dot{Q}$ of Eq.(3), is the sum of the three heat sources applied in the transition layer around the zero-level-set surface $[-\varepsilon,+\varepsilon]$ and the one associated with droplets impingement:

$\dot{Q}=\delta(\varphi)\left[q_{P}+q_{L}-q_{T}\right]+\dot{Q}_{d}$ 


\subsection{Material Supply - Heat input}

The evolution of the metal-gas interface corresponds to the weld bead formation due to the added metal. It results from the mass conservation equation and the momentum equation resolution (ie. Navier-Stokes equation) depending on the external forces and the volume expansion source term, as explained in this section.

\subsubsection{Cavity model for droplets impingement}

It is assumed here, as initially proposed by Lancaster [32], that molten droplets impinge in the fusion zone in a limited cylindrical region. The volume expansion source term corresponds to the added metal rate imposed in the cylinder deduced from the droplet dimensions (Figure 3). This model has later been improved by Kumar and Bhaduri [3]. As detailed by Bellet and Hamide [25], it is considered that each droplet falling in the weld pool generates a cylinder whose radius, $R_{c}$, is twice bigger than its radius, The average droplet radius $R_{d}$ can be easily deduced from the mass flow rate and the frequency of detachment of the droplets, $f_{d}$ :

$R_{d}=\left(\frac{3 \rho_{w}\left(T_{0, w}\right) R_{w}^{2} v_{w}}{4 f_{d} \rho_{w}\left(T_{d}\right)}\right)^{\frac{1}{3}}$

where $\rho_{w}$ is the density of the added metal as previously defined (Eq.(9)), $T_{d}$ is the droplet temperature when impinging the liquid bath. This value is imposed in the calculation and should be deduced from the literature or from experimentations. The detachment frequency $f_{d}$ has to be defined from the GMAW process parameters. It is known to depend on the shielding gas and welding intensity. In the present work, the expression proposed by Kim et al. [16] is used:

$$
f_{d}=\frac{-243.44}{1+\exp \left(\frac{I-291.086}{6.06437}\right)}+323.506-0.8741 I+0.0025 I^{2}
$$

This sigmoid function enables to consider both globular and spray-type transfer domains. $R_{d}$ is then completely defined, and so the radius of the cavity $R_{c}$, which is assumed to be twice as much. As regards the height of the cylinder, $H_{c}$, it is determined by an energy balance involving the kinetic energy of droplets, the hydrostatic pressure in the liquid and the liquid-gas surface tension as suggested by Kumar and Bhaduri [3]:

$$
H_{c}=-\frac{2 \gamma}{R_{c} \rho g}+2 \sqrt{\left(\frac{\gamma}{R_{c} \rho g}\right)^{2}+\frac{R_{c} v_{d}^{2}}{24 g}}
$$

where $\rho$ is the local density of the liquid metal in the fusion zone, $\gamma$ the liquid-gas surface tension, $g$ the gravity and $v_{d}$ the velocity of droplets falling in the weld pool. This velocity can be expressed as:

$v_{d}=\sqrt{v_{d}^{0^{2}}+2 a_{d} L_{a}}$

where $v_{d}^{0}$ is the initial droplet velocity, $a_{d}$ the droplet acceleration assumed constant throughout the arc plasma, and $L_{a}$ the arc length. A constant acceleration can indeed be deduced from the extrapolated graphs of Jones $e t$ al. [3] proposed in [16]. The initial velocity is computed from the equation of Lin et al. [3]:

$v_{d}^{0}=\sqrt{-0.33692+8.54 \times 10^{-6} \frac{I}{2 R_{d}}}$

\subsubsection{Implementation in conservation equations}

As a first approach, only mass and energy equations are supposed to be impacted by material supply. The transfer of momentum from the molten droplets to the liquid in the fusion zone is ignored. This is consistent with the fact that calculation of liquid flow is not a goal in itself in the proposed numerical model. Regarding mass conservation, in the cylindrical region for droplets delivery, the usual equation should include a right hand side 
term which is the ratio of the mass flow rate of filler material $\dot{m}_{w}$ by the volume of the cavity $V_{c}$. This value corresponds to the added metal rate into this cavity. Note that this right hand side term is time averaged (unitary droplets continuously impinge the liquid pool) and space averaged within the delivery region:

$$
\frac{\partial \rho}{\partial t}+\nabla \cdot(\rho \vec{v})=\frac{\dot{m}_{w}}{V_{c}}=\frac{\rho_{w}\left(T_{0, w}\right) R_{w}^{2} v_{w}}{R_{c}^{2} H_{c}}
$$

Neglecting density variations for the liquid, we obtain a simplified equation:

$$
\nabla \cdot \vec{v}=\frac{\dot{m}_{w}}{\rho V_{c}}=\dot{\theta}_{d}=\frac{\rho_{w}\left(T_{0, w}\right)}{\rho} \frac{R_{w}^{2} v_{w}}{R_{c}^{2} H_{c}}
$$

It should be mentioned that the added metal rate calculated in Eq. 19 is considered in the whole domain, without the shrinkage phenomenon occurring during cooling. Thus the added metal will afterwards solidify and its density will increase toward its initial density before melting, $\rho$. Since the shrinkage phenomenon is not considered in the present model, the ratio of density $\rho_{w}\left(T_{0, w}\right) / \rho$ has to be considered equal to 1 in the cavity in order to express the material supply source term. Thus the value of $\dot{\theta}_{d}$ is simplified and expressed as the rate of the volume of wire per unit time as:

$\dot{\theta}_{d}=\left\{\begin{array}{l}\frac{\dot{m}_{w}}{\rho V_{c}}=\frac{R_{w}^{2} v_{w}}{R_{c}^{2} H_{c}} \text { in the cavity } \\ 0 \text { outside the cavity }\end{array}\right.$

Accordingly, the equation for energy conservation should include a right hand side term representing the energy input provided by molten droplets:

$\rho\left(\frac{\partial h}{\partial t}+\vec{v} \cdot \vec{\nabla} h\right)-\nabla \cdot(\lambda \vec{\nabla} T)=\rho \dot{\theta}_{d}\left(h_{d}-h\right)$

where $h_{d}$ denotes the specific mass enthalpy of droplets when delivered: $h_{d}=h\left(T_{d}\right)$. Note that in this equation, the right hand side term is not equal to the droplets heat input rate $\left(\rho \dot{\theta}_{d} h_{d}\right)$. The additional term $\left(-\rho \dot{\theta}_{d} h\right)$ is due to the fact that there exists an external mass (and energy) input: $\nabla \cdot \vec{v} \neq 0$ (Eq. (19)). The details of the calculation steps developed in order to obtain this equation are presented in Annex. In can be noticed that, injecting now Eq.(18) in Eq. (21) we obtain:

$$
\frac{\partial(\rho h)}{\partial t}+\nabla \cdot(\rho h \vec{v})-\nabla \cdot(\lambda \vec{\nabla} T)=\rho \dot{\theta}_{d} h_{d}
$$

In which now, under this form, the right hand side is exactly the heat input rate of the droplets. It can be noted that the right hand side of this advection-diffusion equation is both time and space averaged. Equation (21) corresponds to the conservation equation energy for a specific added metal and energy rates. In the numerical resolution, Eq. (21) will be used with an effective specific heat as explained in Section 2.2.1. , leading to the equivalent equation:

$$
\rho C_{p}\left(\frac{\partial T}{\partial t}+\vec{v} \cdot \vec{\nabla} T\right)-\nabla \cdot(\lambda \vec{\nabla} T)=\rho \dot{\theta}_{d}\left(h_{d}-h\right)
$$

Finally, integrating the surface heat fluxes developed using the CSF method, the heat conservation equation can be written in any location:

$$
\rho C_{p}\left(\frac{\partial T}{\partial t}+\vec{v} \cdot \vec{\nabla} T\right)-\nabla \cdot(\lambda \vec{\nabla} T)=\delta(\varphi)\left[q_{P}+q_{L}-q_{T}\right]+\dot{Q}_{d}
$$


with:

$$
\dot{Q}_{d}=\rho \dot{\theta}_{d}\left(h_{d}-h\right)
$$

\subsection{Weld bead development}

\subsubsection{Material supply modelling}

Regarding the formation of the weld bead shape, the driving forces considered are the normal component of surface tension (proportional to the mean curvature of the gas-metal interface) on one hand and the gravity volume force on the other hand. Arc pressure is not taken into account in the present model. The gravity volume force and the surface tension surface force are expressed as:

$\left\{\begin{array}{l}\vec{T}_{\gamma}=\kappa \gamma \vec{n} \\ \vec{F}_{g}=\rho \vec{g}\end{array}\right.$

where $\kappa$ is the local mean curvature of the interface and $\vec{n}$ is the unit outward normal vector of the interface.

These parameters are computed from the level set function field, $\varphi$ :

$$
\left\{\begin{array}{l}
\kappa=-\nabla \cdot \vec{n} \\
\vec{n}=\frac{\vec{\nabla} \varphi}{\|\vec{\nabla} \varphi\|}
\end{array}\right.
$$

The gravity volume force is applied on the entire liquid domain. Nevertheless the surface tension should be restricted to the liquid-gas interface. This force is transformed in a volume force, $\vec{F}_{\gamma}$, using the Continuum Surface Force method as previously detailed. Consequently the whole volume force considered is:

$\vec{F}=\delta(\varphi) \vec{T}_{\gamma}+\vec{F}_{g}$

Therefore the set of momentum and mass conservation (Eq. (18)-(20)) equations is solved considering a compressible fluid flow:

$$
\left\{\begin{array}{c}
\rho\left(\frac{\partial \vec{v}}{\partial t}+(\vec{v} \cdot \vec{\nabla}) \vec{v}\right)-\nabla \cdot\left(\mu\left(\vec{\nabla} \vec{v}+\vec{\nabla} \vec{v}^{T}\right)\right)+\vec{\nabla} p=\vec{F} \\
\nabla \cdot \vec{v}=\dot{\theta}_{d}
\end{array}\right.
$$

where $p$ is the pressure in the liquid bath and $\mu$ is the enhanced liquid dynamic viscosity, as discussed previously. The compressible aspect of these equations is induced by the material supply source term. Moreover, in no case this set of equations is solved to model the liquid fluid flows since they are not considered here. Lastly, the final velocity $\vec{v}$ is then used as the convective thermal velocity in the energy equation Eq.(3).

\subsubsection{Interface transport}

The liquid velocity corresponding to the added metal rate $\vec{v}$ is specifically used to solve the convection equation of the level set [3]:

$$
\frac{\partial \varphi}{\partial t}+\vec{v} \cdot \vec{\nabla} \varphi=0
$$

The new value of the level set function defines the liquid-metal interface $(\varphi=0)$ at current time regarding the added metal rate. A reinitialization operation is used [3] in order to respect the Eikonal property on the field $\varphi$, 
that is the fact that, as a signed distance function, its gradient should remain unitary (i.e. $\|\nabla \varphi\|=1$ ). This condition is obtained by solving the Hamilton-Jacobi equation:

$$
\frac{\partial \varphi}{\partial \tau}=S(\varphi)(1-|\vec{\nabla} \varphi|)
$$

where $S($.$) is the function sign. More details about the resolution of this equation are available in [3]. Volume$ and energy balances from a 2D material supply model have been carried out in [31] to validate the heat and material supplies models presented up to this point.

\subsection{Numerical resolution}

A two-step algorithm is proposed in order to separate, for each time increment, the computations of the temperature field (Eq. (25)) and of the velocity field for the weld bead development (Eq.(29)). It is illustrated in Figure 5. Implementation details about the numerical resolution can be found in ref. [31]

- The first step is the heat transfer resolution through Eq.(3), in which the velocity field taken into account in the advection term is $\vec{v}$ issued from the resolution of Eq.(29) on the previous time increment

- The second step is the resolution for the velocity $\vec{v}$ resulting from material supply, Eq.(29), with an enhanced value for the liquid viscosity, the surface tension force, and the expansion term as detailed in Eq.(19).

- The third step is the updating of the level set function, using $\vec{v}$, Eq.(30) and (31).

It should be mentioned that an adaptative remeshing has been developed for the welding modeling. Thus an anisotropic remeshing is regularly computed based on metrics deduced from LS position and temperature gradient. As presented before (Figure 2), the FE model includes both metal and gas domains. The interface modelled by the level set function is initially located on a refined anisotropic mesh area surrounding the metalgas interface. At the same time the heat sources move, a refined mesh spot follows them and the interface zone keeps refined. Thus both interface description and weld pool zone are always accurately defined. The constant time step is also chosen as a function of the algorithms. The solvers presented here have been validated, especially in terms of energy and mass conservation. The interested reader can refer to [31] for the details of the various validations developed for the present model. Some metrics have been introduced in order to refine the mesh around the domains of interest where large variations of computed fields are expected: In the thickness of the level set (ie. $\varphi \in[-\varepsilon,+\varepsilon]$ ), in the liquid bath and in both the plasma and laser heat source domains.

\section{Application to the simulation of multipass hybrid laser-arc welding}

\subsection{Geometry, mesh and material properties}

The numerical model proposed in this paper is applied to the simulation of a hybrid laser / GMAW configuration, which is representative of an industrial process, the only change being a reduced length of the studied coupon. As illustrated in Figure 6, a narrow chamfer is filled using a multipass hybrid arc/laser welding process. The chamfer is $50 \mathrm{~mm}$ deep, around $10 \mathrm{~mm}$ wide and the weld bead is made along the $100 \mathrm{~mm}$ length of the chamfer bottom. The filling consists in various layers of two passes each. First, the tandem of heat sources moves along the left side of the centre chamfer axis and then along the right side. Each pass begins from the front part of the workpiece. The initial finite element mesh can be seen in Figure 6. It is composed of 400000 $\mathrm{P} 1+/ \mathrm{P} 1$ tetrahedral elements and 70000 nodes. With a $5.10^{-3} \mathrm{~s}$ time step, a $7 \mathrm{~s}$ welding simulation is completed in 40 hours on 32 AMD Opteron HP cores of $2.36 \mathrm{GHz}$ RAM. Both workpiece and filler materials are identical. The mid-alloyed steel 18MnNiMo5 (ASME SA 533), hereafter called 18MND5 (French AFNOR norm), is used in this configuration. All temperature dependent material properties have been determined by use of the software ThermoCalc from the initial chemical composition of the steel grade. Indeed no macrosegration is considered in the present model. The rank tables are from $20^{\circ} \mathrm{C}$ to $2500^{\circ} \mathrm{C}$. Only the thermal conductivity evolution has been taken from the literature $[29,30]$.

It has not been possible to find in the literature an estimation of the surrounding gas properties, especially in the arc plasma. Consequently the physical properties of the gas have been considered constant and similar to the ones of air. Thus, the phenomenon of the heat evacuation by the gas could be considered.

Table 1 gathers all gas and metal material properties. A temperature dependent continuous value is used in the present model for density, heat capacity and solid thermal conductivity. Only an averaged value is given here for these three parameters. Other parameters are considered as fixed during the whole simulation. The local 
dynamic viscosity is computed as a logarithmic function of the liquid phase fraction in order to continuously evolve between the material properties imposed in solid and liquid phases. A high value is proposed for the solid phase for preventing phase movement (ie. penalisation term). The logarithmic law has to be chosen due to the high difference between the parameters proposed for the two phases. Regarding specific heat, it should be reminded that inside the solidification interval, Eq.(4) is used to determine the effective specific heat in the heat transfer equation.

\subsection{Welding parameters and boundary conditions}

For all welding simulations presented hereafter, process parameters are identical for each pass. These process parameters are listed below:

- The welding velocity is around $1 \mathrm{~m} \cdot \mathrm{min}^{-1}$, the laser beam being located $3 \mathrm{~mm}$ in front of the electrode.

- The power of the laser beam is $5 \mathrm{~kW}$ and the defocused spot has a $3 \mathrm{~mm}$ radius. The laser heat source is considered as a surface Gaussian source since no keyhole phenomenon is considered.

- The GMA torch has a total power of $10 \mathrm{~kW}$ which is then divided into two parts: the first part $(69 \%)$ is used to heat the weld pool and the second one $(31 \%)$ to melt the electrode in the form of droplets. Those values have been computed from the described method of Section 2.2.2.

- The filler metal has a velocity of $10 \mathrm{~m} \cdot \mathrm{min}^{-1}$ and a diameter of $1.2 \mathrm{~mm}$.

Regarding boundary conditions, an ambient temperature, $T_{0}$, equal to $300 \mathrm{~K}$, is applied onto the left, right and upper faces of the model through a Dirichlet condition. Droplets temperature is proposed as equal to $2500 \mathrm{~K}$ according to experimental measures of [38]. This value is in agreement to the global welding simulations (considering all phenomena in both gas and metal domains) achieved by Hu et al. [4, 5] and Fan et al. [39,40]. This droplet temperature value is also in agreement with the above mentioned fraction of $31 \%$ of the total power of the torch. Convective transfer and emissivity are considered on the upper interface between the surrounding gas and the metal: $h_{T}=10 \mathrm{~W} \cdot \mathrm{m}^{-2} \cdot \mathrm{K}^{-1}$ and $\varepsilon_{\mathrm{r}}=0.75$. The high value of this last parameter is proposed in order to enhance the heat exchanges between liquid/solid metal and gas. The initial temperature, $T_{\text {init }}$, of the model is the same as the ambient temperature, $T_{0}$. This temperature is also the one chosen for the filler metal, $T_{0, w}$.

\section{Results \& Discussion}

In order to analyse precisely the efficiency of this three-dimensional transient finite element model, two simulations of this hybrid arc/laser welding process are proposed:

- Case A: The chamfer is filled of two layers (four passes). An enhanced liquid metal thermal conductivity is used as proposed. Consequently, the liquid thermal conductivity given in Table 1 is multiplied by a factor $f_{\lambda}=20$ as considered by Bellet and Hamide [25]. The successive equations solved are, as detailed in Section 2.5. and Figure 5: the energy equation, the mass and momentum equations (material supply and surface tension only) and the level set transport.

- Case B: The choice of a conductivity coefficient $f_{\lambda}$ remains arbitrary. Without any previous optimization work, it is unreal to consider it adapted to the welding parameters. Rather than performing a complex optimization computation, a sensitive study of the conductivity coefficient value on the weld bead geometry is proposed in this paper. Four values of $f_{\lambda}, 1,5,10$ and 20, are considered. Other simulation parameters remain the same as Case A. Only the first pass of the first layer is simulated in this case in order to compare weld bead geometries in different directions.

The differences of weld pool geometry at the end of the first pass from case A and case B are discussed.

\subsection{Case A: Modelling of an hybrid arc / laser multi-pass welding process}

Figure 7 a) shows the weld bead geometry section for the first layer. Numerical simulations (Num) and experimental (Exp) observations are compared. The height and width of the weld beads are correctly predicted on the upper part of the weld. This is shown more clearly on the right hand side pass (Num 2 / Exp 2). The concavity of the surface is also simulated, and is close to the experimental observations for the whole weld bead. The transverse section of the weld bead as an area of $17.1 \mathrm{~mm}^{2}$ which is in agreement with the welding material supply parameters: the difference is close to $0.8 \%$. However the geometry of the solidified domain on the lower part of the weld bead shows a clear difference of the order of a few millimetres. This difference may be explained by the fact that the liquid is static. Indeed no fluid flow is considered. This case implies that the fluid flow does not dig the weld pool, as experimentally observed as a consequence of the Marangoni forces. Figure 7 b) shows the weld bead of the second layer. Same observations can be done on the weld geometry. The concavity of the surface is correctly simulated and the dimensions of the weld bead are also comparable to the 
measurements. Nevertheless the depth of the weld bead shows clear differences on the two passes due to the lack of the fluid flow modelling.

The temperature field is shown on Figure 8 after two seconds of welding on the second layer. The largest temperatures correspond to the position of the electrode. For this position, an average temperature value of 2500 $\mathrm{K}$ is encountered. This value corresponds to the one proposed for the droplets. Consequently droplets impose their own temperature at the surface of the weld pool. Nevertheless these temperatures are lower than the boiling point. This is explained by the large value of the thermal conductivity used in this modelling into the liquid phase. This value leads to a sharp decrease of the temperature gradient especially in the weld pool and increases heat flow through the liquid domain. The temperature field then decreases along the weld bead. Temperatures closed to $500{ }^{\circ} \mathrm{C}$ are obtained after a few seconds of welding on the solidified domains. This temperature field is more precisely defined on Figure 9 in the interface. The development of a large heated and stable domain in the gas domain is shown due to the thermal diffusivity of the gas. This large domain spreads during the whole process. The weld pool is around $1 \mathrm{~cm}$ large and a stable shape is obtained as soon as the process begins.

\subsection{Case B: Sensitive analysis of the conductivity coefficient on the weld bead and weld pool geometry}

The weld bead formation is carried out only for the first pass of the first layer. Four computations are compared, each one with a different conductivity coefficient value. These values increase from $f_{\lambda}=1$ (no conductivity multiplier considered) to Case A conductivity coefficient value, $f_{\lambda}=20$. The intermediate proposed values are $f_{\lambda}=5$ and $f_{\lambda}=10$.

The transversal thermal distribution profiles are gathered on Figure 10. The printed temperature corresponds to the maximal temperature detected by the metal during the welding pass. The purple ring shows the mushy zone. Even though the four weld bead free surfaces are similar, the weld pool spreading are not. Indeed, the use of a multiplier for the liquid conductivity leads to a spreading of the weld pool. The analysis of the mushy zone borders highlights a similarity between the three models with a conductivity coefficient greater than 1 . The width, $w_{T}$, and depth, $d_{T}$, weld pool dimensions are resumed in Table 2 . In the transversal direction, the size of the weld pool is strongly impacted by the use of a conductivity coefficient since the depth is $50 \%$ larger. The width of the weld pool does not significantly change. Figure 10 shows also that the thermal distributions outside the weld pool are close to in the cases b), c) and d). The conductivity multiplier looks to only modify the thermal distribution into the weld pool. This phenomenon was expected as only liquid properties are strongly modified. In fact, the more the liquid conductivity coefficient rises, the more the temperature gradient decreases. Consequently the averaged temperature also decreases from case a) to d). Hence, with a conductivity coefficient $f_{\lambda}=5$, the thermal distribution approaches to case a), $f_{\lambda}=1$.

Most of the optimization analyses performed to compute the right conductivity coefficient are done from experimental macrographics and numerical transversal profiles as in [41]. However, the weld pool geometry also evolves in the welding direction (longitudinal one) according to the coefficient value. Thus a comparison of longitudinal weld bead profiles is necessary.

The longitudinal thermal distribution profiles of the four cases are gathered on Figure 11. The temperature scale is the same as the one of Figure 10. The weld bead has been obtained after $5.5 \mathrm{~s}$ of welding. As expected, the free surface profiles are identical in each case. Only the weld pool dimensions differ. When a conductivity coefficient is used, the weld pool depth, $d_{L}$, rises up of $50 \%$, as before. Moreover, the weld pool length strongly reduces while the mushy zone is stretched in the welding direction. The weld pool length $L_{T L}$, delimited by the liquidus temperature $T_{L}$, and the mushy zone length, $L_{T S-T L}$, delimited by both the solidus and liquidus temperatures (respectively $T_{S}$ and $T_{L}$ ), are resumed in Table 2 . It is interesting to highlight that the mushy zone length remains constant when the conductivity coefficient increases. Only the weld pool length $L_{T L}$ decreases. This phenomenon has to be taken into account while proceeding to an optimization computation. Indeed, additionally to a transversal macrographic comparison, one should compare the length of the experimental and numerical weld pool free surface in steady conditions. This comparison is purchased on Figure 12. Weld pool free surface lengths are resumed in Table 2 for the different values of the conductivity multiplier coefficient. The measured experimental length is $26 \mathrm{~mm}$. The results show that the most appropriate conductivity multiplier is $f_{\lambda}$ $=5$ (Figure $12 \mathrm{~b}$ ). In such conditions the weld pool dimensions are close to experimental observations. Nevertheless, even if the optimization of the conductivity coefficient is carried out, the computed weld pool depth does not reach the experimental one. It may be explained by the lack of fluid flow phenomena in the weld pool.

\section{Conclusion}


A Level Set approach is proposed to model the multipass GMAW / laser hybrid welding. This model couples the resolution of the heat conservation equation, the momentum conservation equation and the weld bead development. The different heat sources developed in this process are precisely modelled. Then a Continuum Surface Force (CSF) approach is applied to impose the whole surface heat sources through the thickness of the Level Set. This model is applied without the fluid flows calculation to decrease computation time. In this simplified approach, an enhanced value of the liquid thermal conductivity is used to model the decrease of the temperature gradient due to fluid flow. The efficiency of this model for multipass hybrid laser / GMAW process simulations has been proved through a comparison of the computed numerical weld beads and experimental macrographics. Without any previous fitting step, the model was able to correctly predict the weld bead development from the first to the fourth pass. Even if none fluid flows modelling was achieved, the numerical width and height of the weld pool were similar to the experimental ones. Nevertheless, it seems inevitable to model the weld pool fluid flows to match the experimental weld pool shape. This should be done considering the momentum sources associated with droplets fall and Marangoni effect. Moreover, a sensitivity study has been carried out to understand how the thermal conductivity coefficient could impact the weld pool geometry. Rather than keep focus on transversal macrographics as mainly done in the literature, the characterization of the thermal conductivity coefficient has been performed comparing the numerical weld pool free surface length to the one measured from experimental weld pool surface pictures.

\section{Acknowledgement}

This work is currently supported by the French Agence Nationale de la Recherche (ANR) in the framework of the project SISHYFE (ANR-09-MAPR-0019). The authors would like to thank the 'Institut Carnot de Bourgogne' (ICB), Le Creusot (France) for the present experimental observations.

\section{Annex}

For any subdomain $\omega$ of the current system $\Omega$, the energy conservation writes, from the first principle of thermodynamics:

$$
\frac{\mathrm{d}}{\mathrm{d} t}\left(\int_{\omega} \rho\left(e+\frac{1}{2} \mathbf{v}^{2}\right) \mathrm{d} V\right)=\int_{\omega} \rho \mathbf{f} \cdot \mathbf{v} \mathrm{d} V+\int_{\partial \omega} \mathbf{T} \cdot \mathbf{v} \mathrm{d} S+\int_{\omega} r \mathrm{~d} V+\int_{\partial \omega} q \mathrm{~d} S
$$

with $\rho$ the density, $e$ the internal energy per unit of mass, $\mathbf{v}$ the velocity field, $\mathbf{f}$ the mass density of external forces, $\mathbf{T}$ the external surface forces, $r$ the volume density of heat input and $q$ the surface density of heat input.

For the left hand side tem, in a context of condensed matter at quasi-constant pressure, the internal energy density $e$ and the specific enthalpy $h$ per unit mass $(i . e . h=e+p / \rho)$ can be considered identical.

For the right hand side terms in Eq. A1, we can do some developments. Firstly, $q$ is associated with the heat exchange by conduction. Using Fourier law, the heat flux vector is equal to $\mathbf{q}=-\lambda \nabla T$ and the expression of $q$ is $q=\lambda \nabla T$.n. The divergence theorem can then be applied in order to transform the surface integral into a volume integral. Secondly, $r$ is associated either with radiation (in volume), chemical reactions or Joule effect. In the present model, $r$ is equal to 0 . Indeed, the heat input per unit volume into the system is due to the added metal. Finally, using the theorem of kinetic energy, the two first integrals of the right hand side of Eq. (A1) can also be transformed and expressed with the volume integral of the deformation power and the total time derivative of the velocity.

These mathematical developments lead finally to:

$$
\frac{\mathrm{d}}{\mathrm{d} t}\left(\int_{\omega} \rho\left(h+\frac{1}{2} \mathbf{v}^{2}\right) \mathrm{d} V\right)=\int_{\omega} \boldsymbol{\sigma}: \dot{\boldsymbol{\varepsilon}} \mathrm{d} V+\int_{\omega} \rho \frac{\mathrm{d} \mathbf{v}}{\mathrm{d} t} \cdot \mathbf{v} \mathrm{d} V+\int_{\omega} \nabla(\lambda \nabla T) \mathrm{d} V
$$

The deformation power in the weld pool, due to very weak dynamic viscosity can be neglected. Consequently, in any point of the domain the double dot product $\boldsymbol{\sigma}: \dot{\boldsymbol{\varepsilon}}$ is equal to 0 . 
On another hand Eq. (A2) should also be solved considering the mass conservation equation expressed by:

$\frac{\mathrm{d}}{\mathrm{d} t}\left(\int_{\omega} \rho \mathrm{d} V\right)=0$

Let us consider now the context of a subdomain $\omega$ of variable mass and energy. This is the context of the present study, in which some regions of the fusion zone are submitted to both mass and energy inputs associated with the fall of droplets. In such a context, as discussed in [42], Eq. (A2) and (A3) must be complemented by the rate of variation of energy and the rate of variation of mass, respectively.

The equation for mass conservation (Eq. A3) then becomes:

$\frac{\mathrm{d}}{\mathrm{d} t}\left(\int_{\omega} \rho \mathrm{d} V\right)=\frac{\delta m_{\omega}}{\delta t}$

with $\delta m_{\omega} / \delta t$ the rate of mass input in $\omega$. Denoting $\dot{Q}_{m}$ its volume density, we have:

$\frac{\mathrm{d}}{\mathrm{d} t}\left(\int_{\omega} \rho \mathrm{d} V\right)=\int_{\omega} \dot{Q}_{m} \mathrm{~d} V$

The total time derivative of the integral in the left hand side can be transformed by applying a well-known result [43]:

$\frac{\mathrm{d}}{\mathrm{d} t}\left(\int_{\omega} \rho \mathrm{d} V\right)=\int_{\omega}\left(\frac{\mathrm{d} \rho}{\mathrm{d} t}+\rho \nabla \cdot \mathbf{v}\right) \mathrm{d} V$

Equations (A5) and (A6) lead to the following partial differential equation in the context of mass supply, which corresponds to Eq. (18) in the present paper:

$\frac{\mathrm{d} \rho}{\mathrm{d} t}+\rho \nabla \cdot \mathbf{v}=\frac{\partial \rho}{\partial t}+\nabla \cdot(\rho \mathbf{v})=\dot{Q}_{m}$

Regarding now the energy equation, Eq. (A2), we first complement it with $\delta E_{\omega} / \delta t$ the rate of energy input in $\omega$, associated with the energy transfer through the droplets.

$\frac{\mathrm{d}}{\mathrm{d} t}\left(\int_{\omega} \rho\left(h+\frac{1}{2} \mathbf{v}^{2}\right) \mathrm{d} V\right)=\int_{\omega} \rho \frac{\mathrm{d} \mathbf{v}}{\mathrm{d} t} \cdot \mathbf{v} \mathrm{d} V+\int_{\omega} \nabla \cdot(\lambda \nabla T) \mathrm{d} V+\frac{\delta E_{\omega}}{\delta t}$

Denoting $\dot{Q}_{e}$ the volume density of energy input $\left(\mathrm{W} \mathrm{m}^{-3}\right)$, and applying the rule for total time derivation of the integral in the left hand side, we have:

$\int_{\omega}\left(\frac{\mathrm{d}}{\mathrm{d} t}\left(\rho\left(h+\frac{1}{2} \mathbf{v}^{2}\right)\right)+\rho\left(h+\frac{1}{2} \mathbf{v}^{2}\right) \nabla \cdot \mathbf{v}\right) \mathrm{d} V=\int_{\omega} \rho \frac{\mathrm{d} \mathbf{v}}{\mathrm{d} t} \cdot \mathbf{v} \mathrm{d} V+\int_{\omega} \nabla \cdot(\lambda \nabla T) \mathrm{d} V+\int_{\omega} \dot{Q}_{e} \mathrm{~d} V$

This yields the following local partial derivative equation:

$\frac{\mathrm{d}}{\mathrm{d} t}\left(\rho\left(h+\frac{1}{2} \mathbf{v}^{2}\right)\right)+\rho\left(h+\frac{1}{2} \mathbf{v}^{2}\right) \nabla \cdot \mathbf{v}=\rho \frac{\mathrm{d} \mathbf{v}}{\mathrm{d} t} \cdot \mathbf{v}+\nabla \cdot(\lambda \nabla T)+\dot{Q}_{e}$

or by developing the total derivative:

$\left(h+\frac{1}{2} \mathbf{v}^{2}\right)\left(\frac{\mathrm{d} \rho}{\mathrm{d} t}+\rho \nabla \cdot \mathbf{v}\right)+\rho \frac{\mathrm{d} h}{\mathrm{~d} t}=\nabla \cdot(\lambda \nabla T)+\dot{Q}_{e}$

Injecting Eq. (A7) in this equation leads to: 


$$
\left(h+\frac{1}{2} \mathbf{v}^{2}\right) \dot{Q}_{m}+\rho \frac{\mathrm{d} h}{\mathrm{~d} t}=\nabla \cdot(\lambda \nabla T)+\dot{Q}_{e}
$$

In the context of the present study, the kinetic energy is neglected for the flow in the fusion zone. Thus the square of the velocity can be neglected compared to the local value of the specific enthalpy. This stands also for the kinetic energy transferred from the droplets to the weld pool. Consequently the term $\dot{Q}_{e}$ is restricted to the internal energy of the droplets, corresponding to an enthalpy $h_{d}$. This leads to $\dot{Q}_{e}=h_{d} \dot{Q}_{m}$ and:

$\rho \frac{\mathrm{d} h}{\mathrm{~d} t}=\rho\left(\frac{\partial h}{\partial t}+\nabla h \cdot \mathbf{v}\right)=\nabla \cdot(\lambda \nabla T)+h_{d} \dot{Q}_{m}-h \dot{Q}_{m}$

Finally we get:

$\rho\left(\frac{\partial h}{\partial t}+\nabla h \cdot \mathbf{v}\right)-\nabla \cdot(\lambda \nabla T)=h_{d} \dot{Q}_{m}-h \dot{Q}_{m}$

which is Eq. (21) of the present paper, with:

$\dot{Q}_{m}=\frac{\dot{m}_{w}}{V_{c}}=\rho \dot{\theta}_{d}$ 


\section{References}

[1] R.T.C. Choo, J. Szekely, R.C. Westhoff, On the calculation of the free surface temperature of gastungsten-arc weld pools from first principles: Part i. modelling the welding arc. Metallurgical Transactions B, 23 (1992) 357-369.

[2] R.T.C. Choo, J. Szekely, S.A. David, On the calculation of the free surface temperature of gas-tungstenarc weld pools from first principles: Part ii. modelling the weld pool and comparison with experiments. Metallurgical Transactions B, 23 (1992) 371-384.

[3] H. G. Fan, H. L. Tsai, and S. J. Na, Heat transfer and fluid flow in a partially or fully penetrated weld pool in gas tungsten arc welding. International Journal of Heat and Mass Transfer, 44 (2001) 417-428.

[4] J. Hu, H.L. Tsai, Heat and mass transfer in gas metal arc welding. part i: The arc. International Journal of Heat and Mass Transfer, 50 (2007) 833-846.

[5] J. Hu, H.L. Tsai, Heat and mass transfer in gas metal arc welding. part ii: The metal. International Journal of Heat and Mass Transfer, 50 (2007) 808-820.

[6] G. Xu, J. Hu, H.L. Tsai, Three-dimensional modelling of arc plasma and metal transfer in gas metal arc welding. International Journal of Heat and Mass Transfer, 52 (2009) 1709-1724.

[7] F. Lu, S. Yao, S. Lou, Y. Li, Modeling and finite element analysis on gtaw arc and weld pool. Computational Materials Science, 29 (2004) 371-378.

[8] A. Traidia, F. Roger, Numerical and experimental study of arc and weld pool behaviour for pulsed current gta welding. International Journal of Heat and Mass Transfer, 54 (2011) 2163-2179.

[9] K. Hong, D.C. Weckman, A.B. Strong, W. Zheng, Modelling turbulent thermofluid flow in stationary gas tungsten arc weld pools. Science and Technology of Welding and Joining, 7 (2002) 125-136.

[10] J. Jaidi, P. Dutta, Three-dimensional turbulent weld pool convection in gas metal arc welding process. Science and Technology of Welding and Joining, 9 (2003) 407-414.

[11] N. Chakraborty, S. Chakraborty, Modelling of turbulent molten pool convection in laser welding of a copper / nickel dissimilar couple. International Journal of Heat and Mass Transfer, 50 (2007) 1805-1822.

[12] B. Ribic, R. Rai, T. Debroy, Numerical simulation of heat transfer and fluid flow in gta/laser hybrid welding. Science and Technology of Welding \& Joining, 13 (2008) 683-693.

[13] C.S. $\mathrm{Wu}$, K.C. Tsao, Modelling the three-dimensional fluid flow and heat transfer in a moving weld pool. International Journal for Computer-Aided Engineering, 7 (1990) 241-248.

[14] A. Kumar, T. DebRoy, Heat transfer and fluid flow during gas-metal-arc fillet welding for various joint configurations and welding positions. Metallurgical and Materials Transactions A, 38 (2007) 506-519.

[15] G. Xu, C. Wu. Numerical analysis of weld pool geometry in globular-transfer gas metal arc welding. Frontiers of Materials Science in China, 1 (2007) 24-29.

[16] C.-H. Kim, W. Zhang, T. DebRoy, Modelling of temperature field and solidified surface profile during gas / metal arc fillet welding. Journal of Applied Physics, 94 (2003) 2667-2679.

[17] A. Traidia. Multiphysics modelling and numerical simulation of GTA weld pools. PhD thesis, Ecole polytechnique, 2011.

[18] H. Ki, J. Mazumder, P. Mohanty, Modelling of laser keyhole welding: Part i. mathematical modelling, numerical methodology, role of recoil pressure, multiple reflections, and free surface evolution. Metallurgical and Materials Transactions A, 33 (2002) 1817-1830.

[19] H. Ki, J. Mazumder, P. Mohanty, Modelling of laser keyhole welding: Part ii. simulation of keyhole evolution, velocity, temperature profile, and experimental verification. Metallurgical and Materials Transactions A, 33 (2002) 1831-1842.

[20] H. Zhao, T. Debroy, Macroporosity free aluminum alloy weldments through numerical simulation of keyhole mode laser welding. Journal of Applied Physics, 93 (2003) 10089-10097.

[21] R. Rai, S.M. Kelly, R. P. Martukanitz, T. DebRoy, A convective heat-transfer model for partial and full penetration keyhole mode laser welding of a structural steel. Metallurgical and Materials Transactions, 39 (2008) 98-112.

[22] T. Zhang, C.S. Wu, G.L. Qin, X.Y. Wang, S.Y. Lin, Thermomechanical analysis for laser gmawp hybrid welding process. Computational Materials Science, 47 (2010) 848-856.

[23] R. Wang, Y Lei, Y Shi, Numerical simulation of transient temperature field during laser keyhole welding of 304 stainless steel sheet. Optics \& Laser Technology, 43 (2011) 870-873.

[24] J.U. Brackbill, D.B. Kothe, C. Zemach, A continuum method for modelling surface tension. Journal of Computational Physics, 100 (1991) 335-354.

[25] M. Bellet and M. Hamide. Direct modelling of material deposit and identification of energy transfer in gas metal arc welding. International Journal of Numerical Methods for Heat \& Fluid Flow, 8 (2013) 1340-1355. 
[26] K. Morgan, R.W. Lewis, O.C. Zienkiewicz, An improved algorithm for heat conduction problems with phase change. International Journal for Numerical Methods in Engineering, 12 (1978) 1191-1195.

[27] G.H. Gulliver, The quantitative effect of rapid cooling upon the constitution of binary alloys. Journal of the Institute of Metals, 3 (1915) 262-291.

[28] E. Scheil. Bemerkungen zur schichtkristallbildung, Zeitschrift für Metallkunde, 34 (1942) 70-72.

[29] M. Coret, S. Calloch, A. Combescure, Experimental study of the phase transformation plasticity of 16mnd5 low carbon steel under multiaxial loading. International Journal of Plasticity, 18 (2002) 1707-1727.

[30] P. Dupas, F. Waeckel, Recueil bibliographique de caractéristiques thermomécaniques pour l'acier de cuve, les revêtements inoxydables et les alliages 182 et 600. Technical report, EDF, DER-IMA (Châtou, France), 1994.

[31] O. Desmaison. Modélisation numérique d'un procédé de soudage hybride arc / laser en approche level set : application au soudage multi-passes de tôles d'acier de forte épaisseur. PhD thesis, Mines ParisTech, 2013.

[32] J.F. Lancaster, The physics of welding. Physics in technology, 15, 1984.

[33] S. Kumar, S.C. Bhaduri, Three-dimensional finite element modelling of gas metal-arc welding, Metallurgical Transactions B, 25 (1994) 435-441.

[34] L. A. Jones, T. T. W. Eagar, J. H Lang, A dynamic model of drops detaching from a gas metal arc welding electrode. Journal of physics D: Applied physics, 31 (1998) 107-123.

[35] Q. Lin, X. Li, S.W. Simpson. Metal transfer measurements in gas metal arc welding. Journal of Physics D: Applied Physics, 34 (2001) 347-353.

[36] L. Ville, L. Silva, T. Coupez. Convected level set method for the numerical simulation of fluid buckling. International Journal for Numerical Methods in Fluids, 66 (2011) 324-344.

[37] O. Basset, Simulation Numerique d'Ecoulements Multi Fluides sur Grille de Calcul. PhD thesis, Mines ParisTech, 2006.

[38] G. Jelmorini, G.W. Tichelaar, and G.J.P.M. van den Heuvel. Droplet temperature measurements in arc welding. Technical report, International Institute of Welding, 1977.

[39] H. G. Fan, R. Kovacevic, Droplet formation, detachment, and impingement on the molten pool in gas metal arc welding. Metallurgical and Materials Transactions B, 30 (1999) 791-801.

[40] H G Fan, R Kovacevic, A unified model of transport phenomena in gas metal arc welding including electrode, arc plasma and molten pool. Journal of Physics D: Applied Physics, 37 (2004) 2531-2544.

[41] M. Hamide, Modélisation numérique du soudage à l'arc des aciers. PhD thesis, Mines ParisTech, 2008.

[42] P. Germain, Mécanique, Tome 1, Ecole Polytechnique, Ellipses (1986)

[43] J. Salençon, Mécanique des milieux continus, Tome1, Concepts généraux, Ellipses, Paris (1988) ISBN 2-7298-8854-3 [accessible on the internet at: www.editions.polytechnique.fr/files/pdf/EXT_1245_0.pdf] 


\section{Figures:}

Figure 1: Hybrid laser - GMAW model description according to the governing equations: schematic industrial process (a), energy equation model (b), material supply model (c).

Figure 2: The meshed gas-metal interface and corresponding Level Set function. Level Set function $\varphi$ is considered as equal to 0 on the boundary between gas and metal which is shown by the bold black line. The transition domain of the Heaviside function is located between the two purple bold lines corresponding to the positions $-\varepsilon$ and $+\varepsilon$.

Figure 3: Heat sources of the hybrid laser / GMAW process: $\dot{Q}_{L}$, laser beam heat source, $\dot{Q}_{P}$, plasma heat source, $\dot{Q}_{D}$, droplets heat source and $\dot{Q}_{T}$, heat transfer at the gas / metal interface.

Figure 4: Laser and plasma heat source geometry with the angular Gaussian condition. Laser heat source is vertical and located at the infinite. Point $\mathrm{M}_{\mathrm{L}}$ is located in the laser heat source domain. $\alpha$ is the half-angle of the plasma heat source. Point $\mathrm{M}_{\mathrm{P}}$ is locally defined with the distance $d$ from the plasma origin, $\mathrm{O}$, and with the angular position, $\beta$.

Figure 5: Schematic of the general algorithm proposed to model the hybrid Laser-GMAW process in a Level Set approach. Superscript $t$ and $t+\Delta t$ refer to the value of the fields at computation time $t$ and $t+\Delta t$.

Figure 6: Description of the welding simulation: a) Geometry b) Finite element mesh in a Level Set approach. Both chamfer and metal sample are meshed as proposed in the present model.

Figure 7: Comparison of the weld bead geometry in a cross section of the chamfer for a) the first layer and b) the second layer. Words "Num" and "Exp" respectively refer to the numerical simulations and experimental observations.

Figure 8: Temperature field during the simulation of the third pass, corresponding to the first part of the second layer. The scheme shows the superimposition of the three passes. White lines represent the iso-fraction curves for the solid fraction.

Figure 9: Advancement of the weld pool during the first pass for two process times a) $3.5 \mathrm{~s}$ and b) $5 \mathrm{~s}$. Temperature and liquid fraction fields are respectively shown on the upper part and lower part of the two figures. c) Weld development shown on the back side of the chamfer with the middle cutting plane. The purple color defines the mushy domain corresponding to the temperature interval between solidus and liquidus lines.

Figure 10: Transversal temperature distribution profile of the first pass of welding. Four values of conductivity coefficient: a) 1, b) 5, c) 10 and d) 20.

Figure 11: Longitudinal temperature distribution profile of the first pass of welding. Four values of conductivity coefficient: a) 1, b) 5, c) 10 and d) 20.

Figure 12: Experimental weld pool surface picture a) and surface temperature distribution of the first pass of welding for four values of conductivity coefficient: b) 1, c) 5, d) 10 and e) 20 . Length of weld pool is indicated above simulations. 

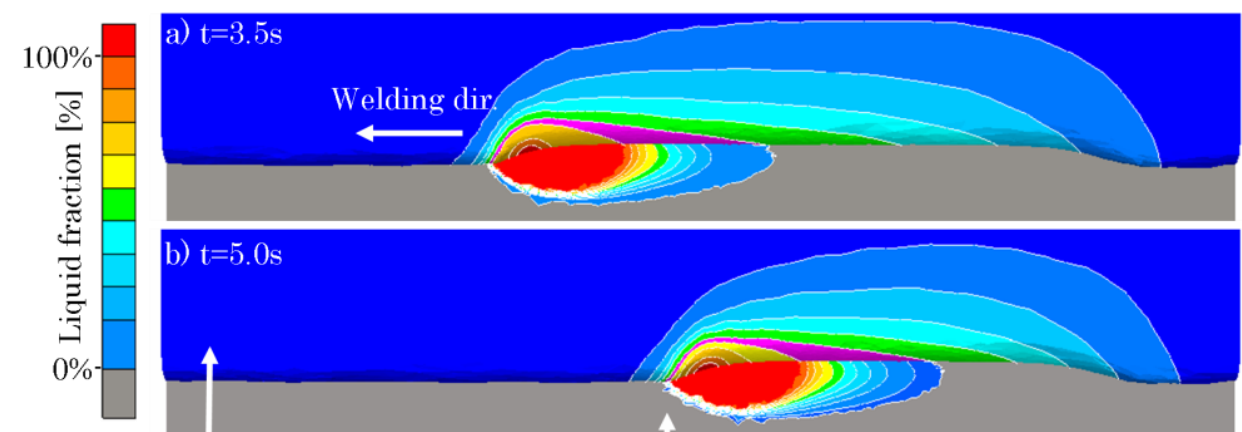

\section{b) $\mathrm{t}=5.0 \mathrm{~s}$}

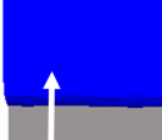

Interface (temperature field)

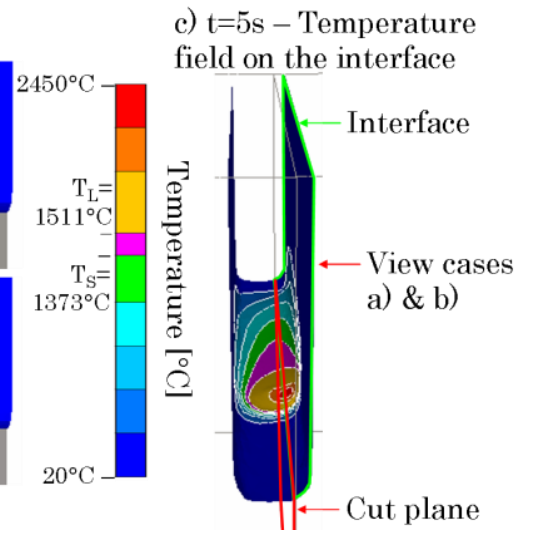

Figure 1 


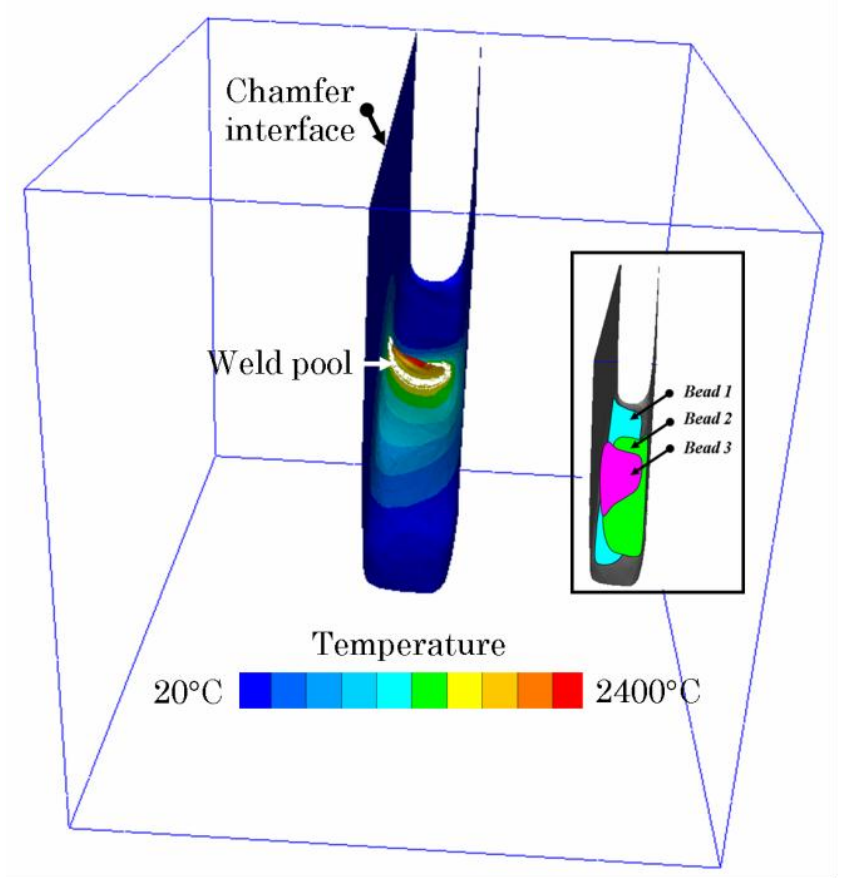

Figure 2 

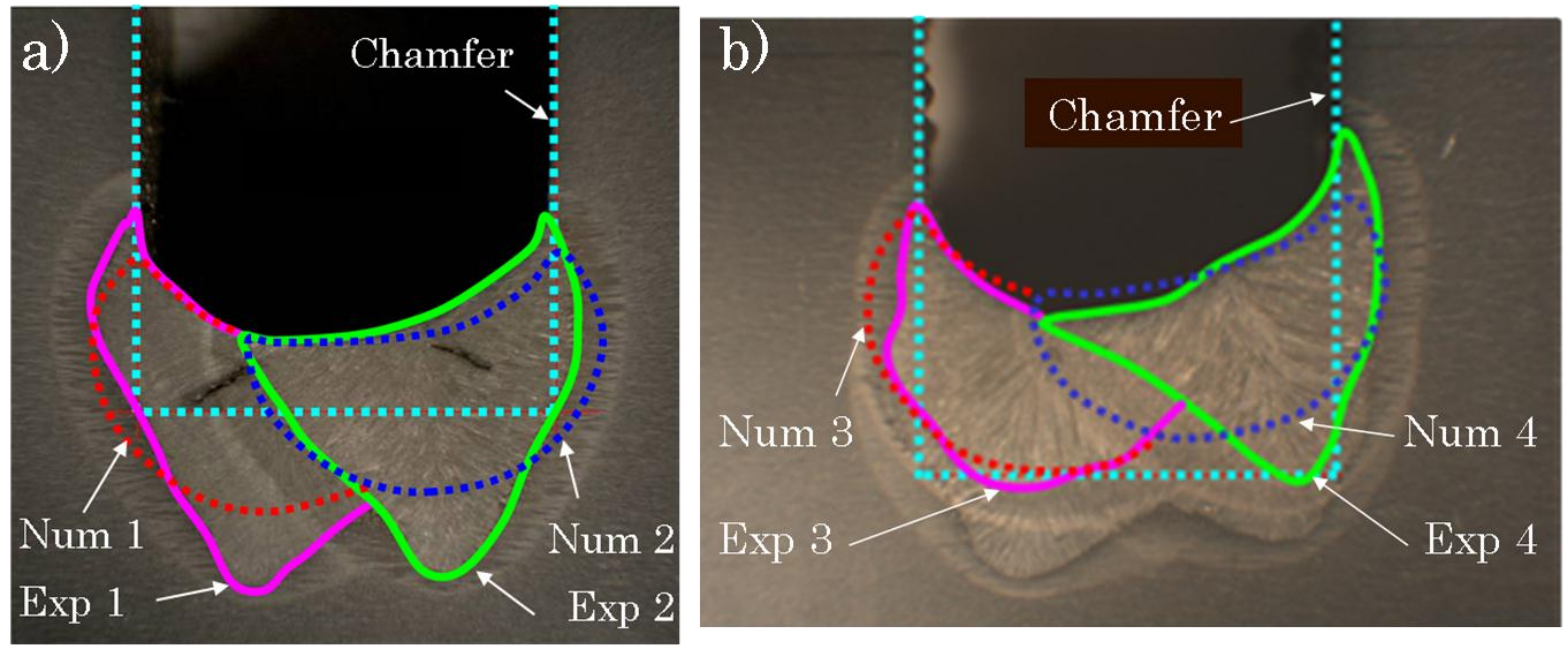

Figure 3 

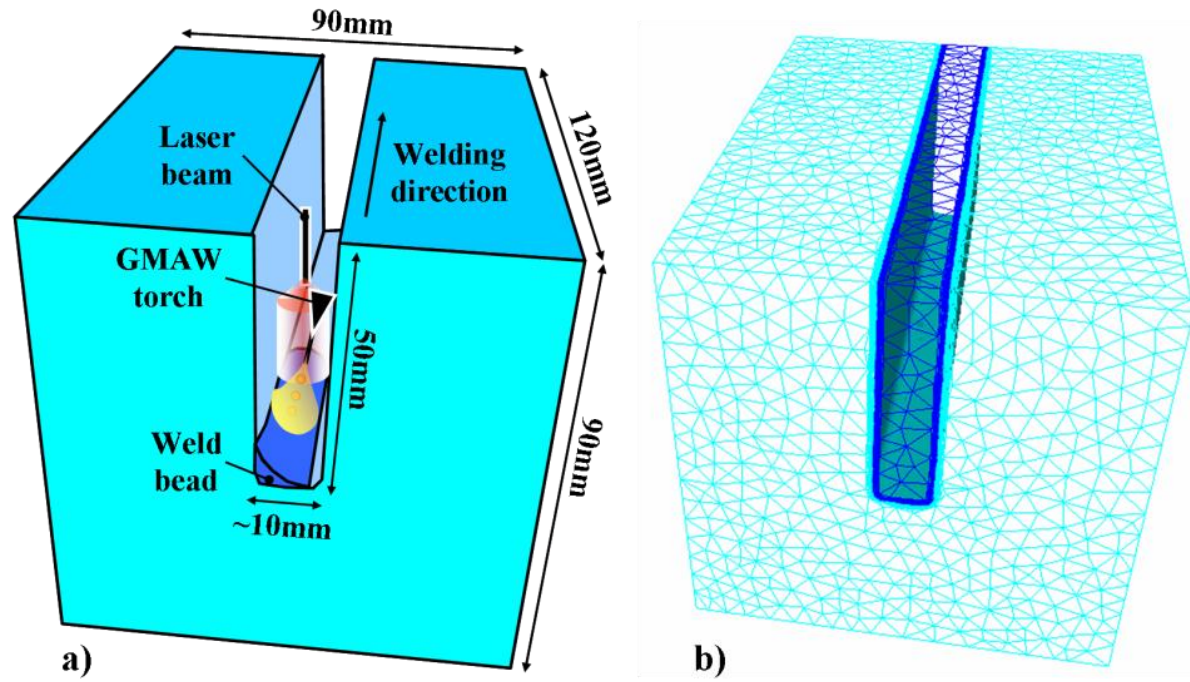

Figure 4 


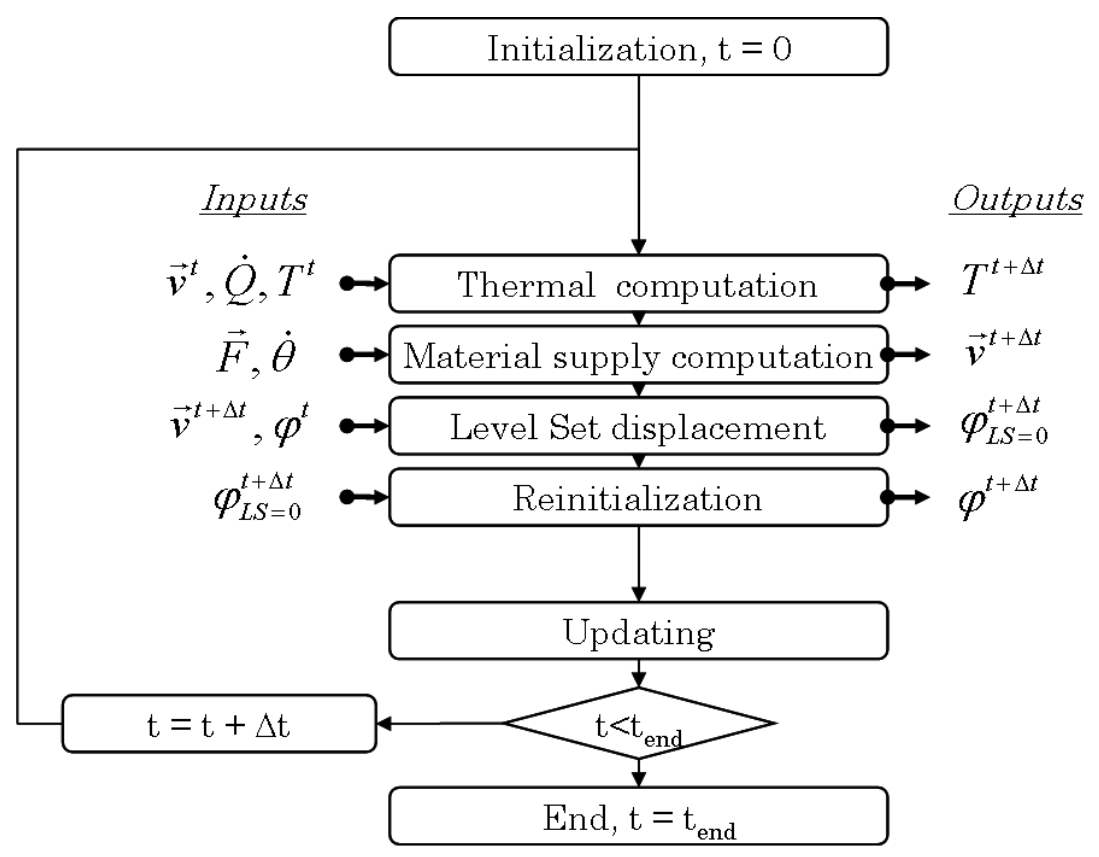

Figure 5 


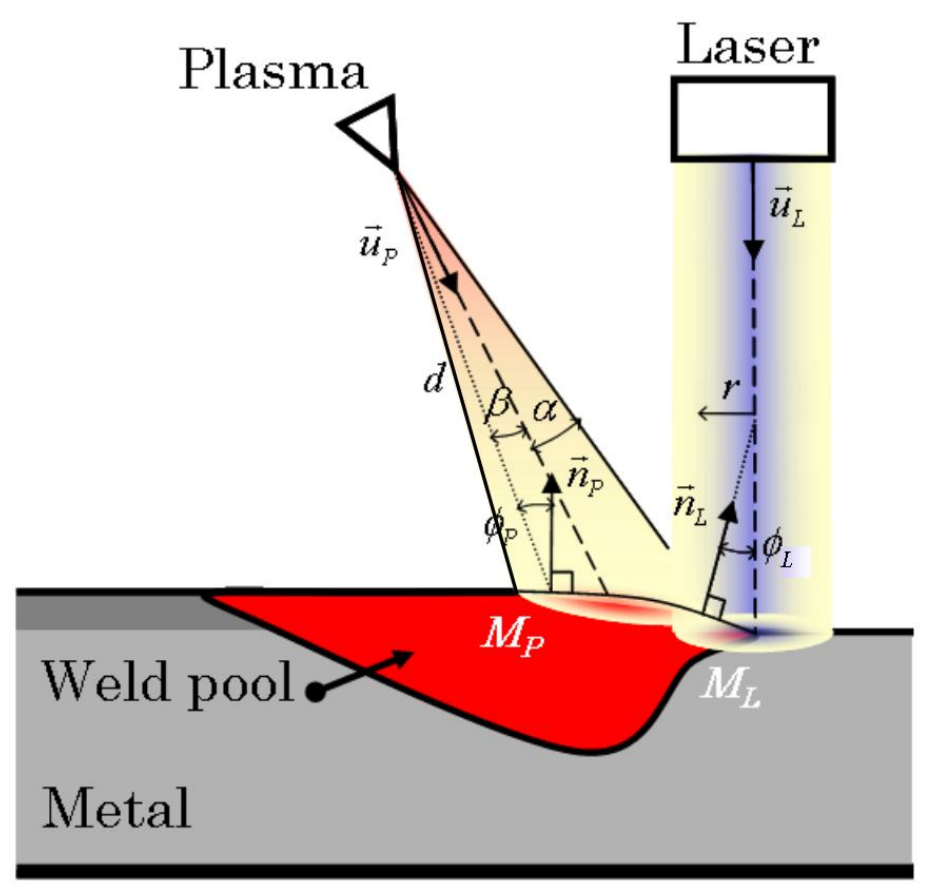

Figure 6 


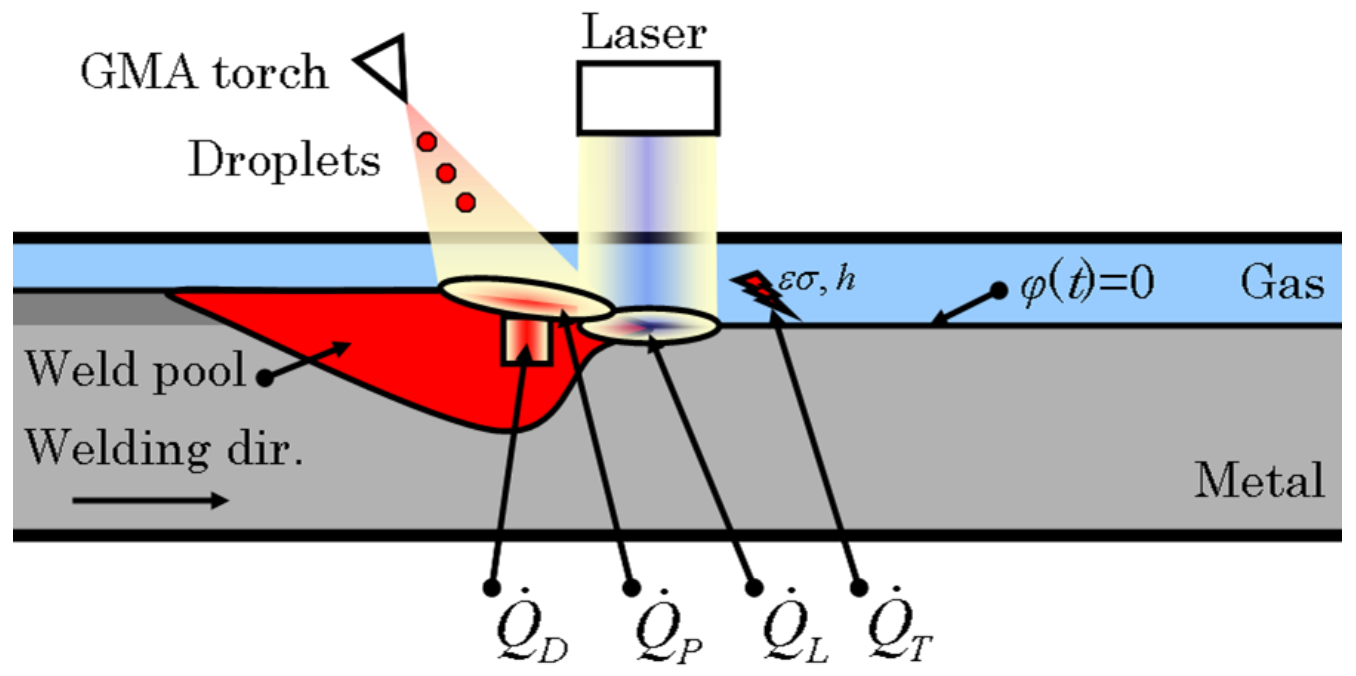

Figure 7 


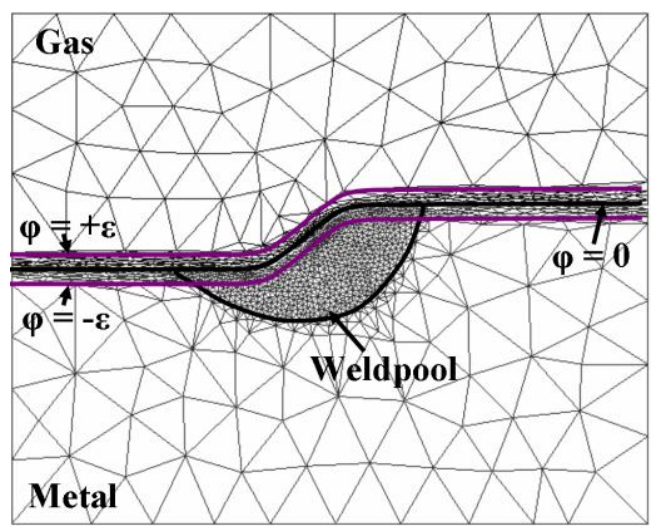

Figure 8 

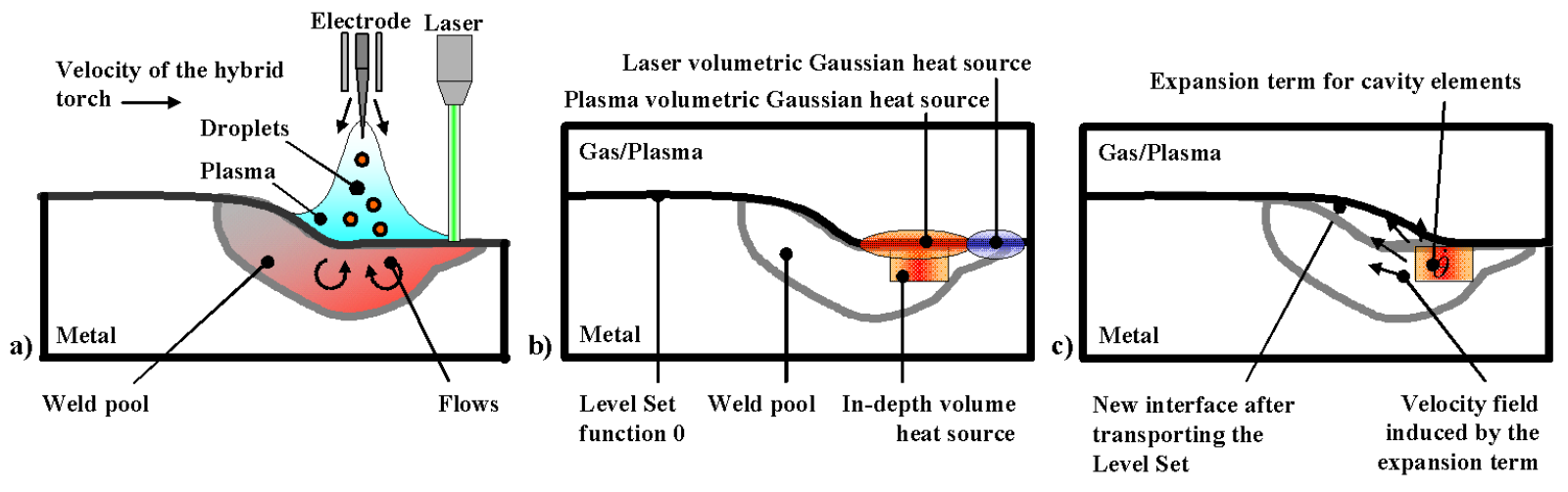

Figure 9 

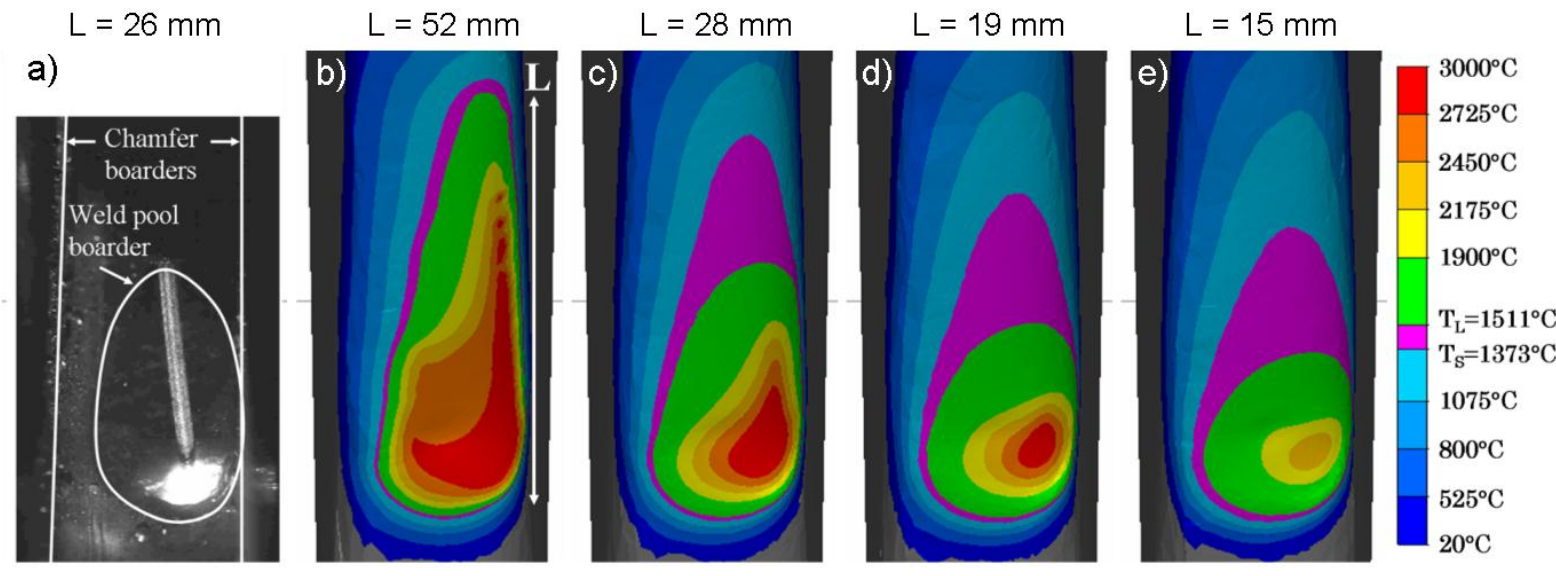

Figure 10 


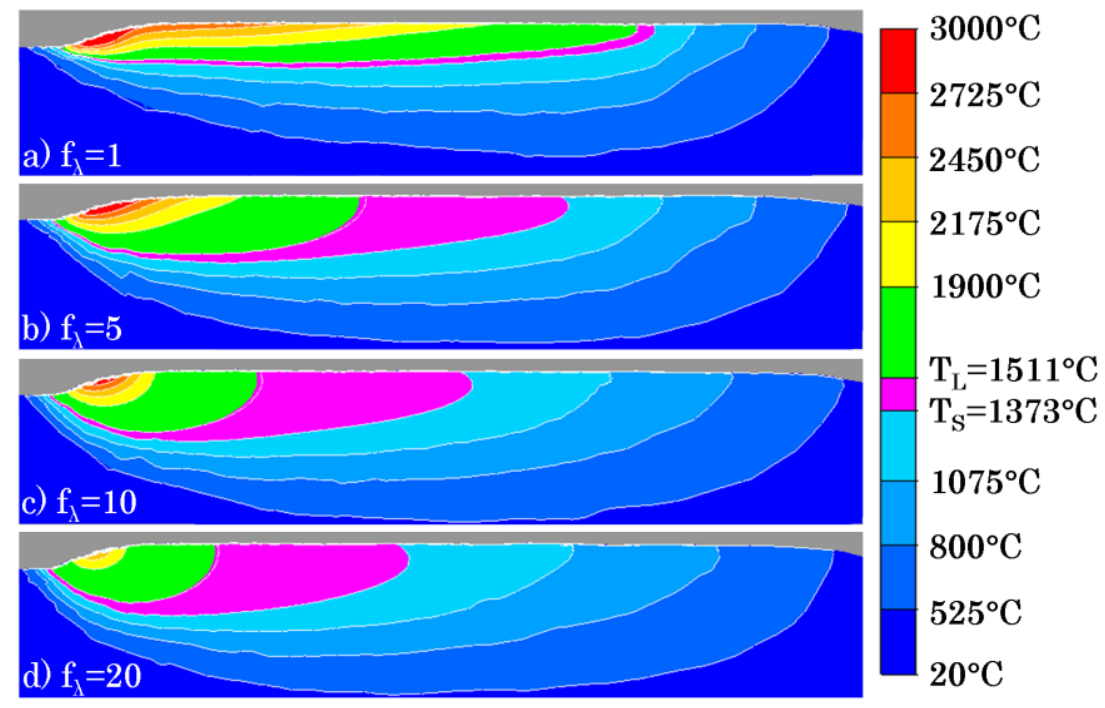

Figure 11 


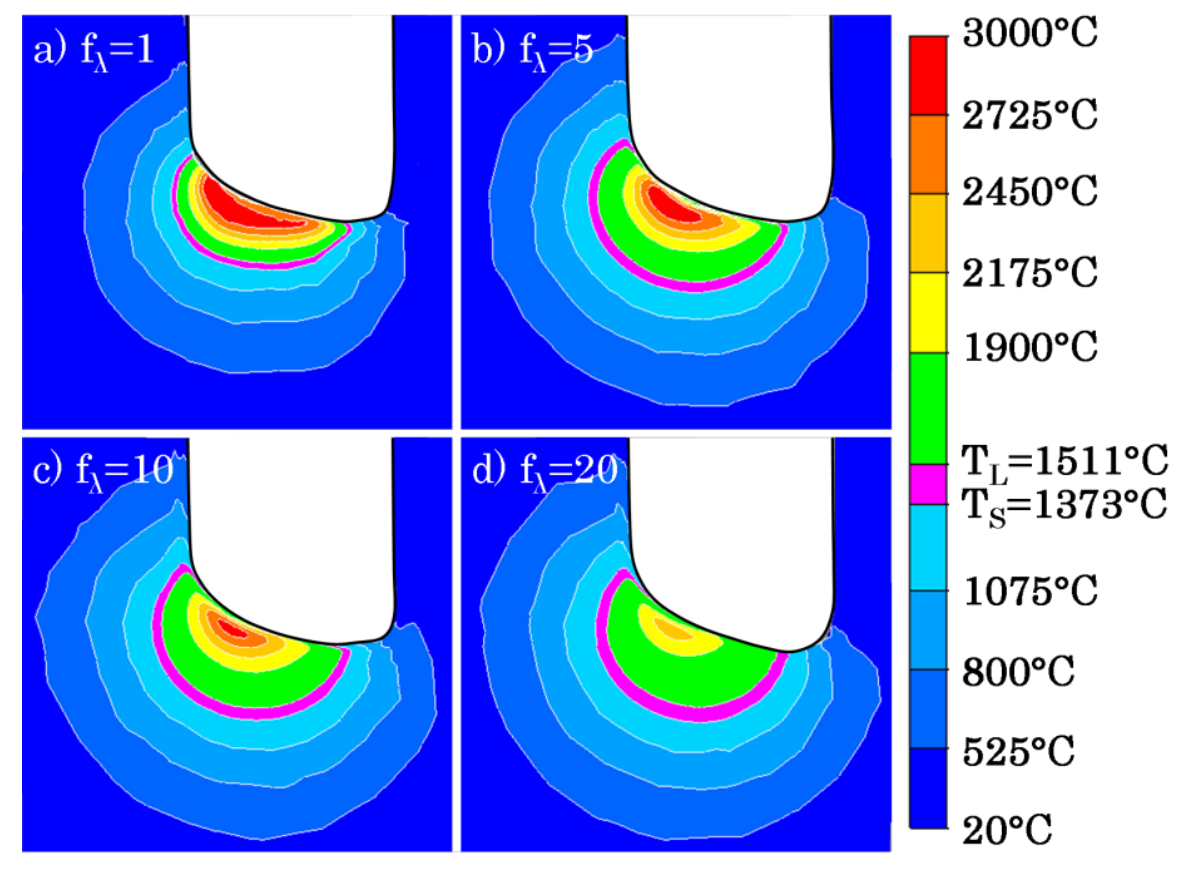

Figure 12 